Journal of Non-Newtonian Fluid Mechanics, 3 (1977/1978) 281-287

(C) Elsevier Scientific Publishing Company, Amsterdam - Printed in The Netherlands

Short Communication

\title{
THE MAXIMUM AMPLITUDE OF STRAIN FOR THE VALIDITY OF LINEAR VISCOELASTICITY
}

\section{GIANNI ASTARITA}

Istituto di Principii di Ingegneria Chimica, Università di Napoli (Italy)

and R.J.J. JONGSCHAAP

Department of Applied Physics, Twente University of Technology, Enschede (The Netherlands)

(Received July 29, 1977)

\section{Introduction}

It is well known that experimental data on samples of non-linearly viscoelastic materials subjected to oscillatory strain of sufficiently small amplitude can be interpreted on the basis of linear viscoelasticity theory. Indeed, Coleman and Noll [1] have shown that the theory of linear viscoelasticity has a proper asymptotic status within the general theory of simple materials with fading memory. Furthermore, most, if not all, constitutive equations which have been discussed in the literature for non-linear viscoelastic materials degenerate into some special form of linear viscoelasticity for oscillatory motions of sufficiently small amplitude and/or frequency.

The question addressed in this paper is the following one: what is, in general, the maximum amplitude of oscillatory strain allowable if linear viscoelasticity theory has to be obeyed to within a preassigned degree of approximation? And, more crucially, does this maximum amplitude of strain depend on the value of the imposed frequency or does it not?

The problem to be discussed is related to a classification of constitutive equations which has been discussed by Marrucci and Astarita [2]; they have shown that a rather large category of constitutive equations which have appeared in the literature do not enjoy the smoothness properties embodied in the theory of simple materials with fading memory, and do not degenerate into linear viscoelasticity in what is considered the proper asymptotic limit.

Available experimental evidence [3,4] seems to indicate that the maximum allowable strain $\psi_{o r}$ is independent of the imposed frequency. At a recent meeting, however, experimental evidence obtained with an eccentric rotating 
disk viscometer was presented [5], showing that $\psi_{\mathrm{cr}}$ increases with decreasing frequency at very low frequencies, while it is constant at large frequencies. The same authors also presented a simplified analysis, showing that such behavior is to be considered typical of simple materials with fading memory.

\section{Physical statement of the problem}

In this section, a simple physical picture of the problem considered is presented, from which the main results of this work can be deduced by intuition.

A simple material with fading memory may be regarded as one where the stress at any one time depends on the strains imposed at all times prior to, and including, the instant of observation. The memory of the material is fading in the sense that strains imposed in the "distant" past have less influence on the present stress than those imposed in the "recent" past. In order to obtain a very crude picture, one may think that the material forgets the distant past and remembers only the recent past. Of course, "distant" and "recent" need to be understood with respect to some appropriate yardstick for the memory span of the material, say with respect to some "natural time" $\Lambda$ of the material.

Now suppose such a material is subjected to an oscillatory strain of amplitude $\psi$ and frequency $\omega$. Consider first the case where $\omega \Lambda>>1$, so that the material "remembers" more than one cycle of strain. Under such conditions, past strains will appear as "small" to the material only if the amplitude of strain $\psi$ is small enough; hence, one would expect the maximum allowable strain $\psi_{\mathrm{cr}}$ to be independent of the frequency $\omega$.

Conversely, consider the case where $\omega \Lambda<<1$, so that the material only remembers a small fraction of a cycle, in fact a fraction equal to $\omega \Lambda$. Under such conditions, past strains (relative to the present configuration, as appropriate if fluid materials are considered) will appear as small to the material provided they are small over a fraction $\omega \Lambda$ of a cycle. Since the strains imposed over such a fraction are no larger than $\omega \Lambda \psi$ (where $\psi$ is the amplitude of strain over the whole cycle), one would expect $\psi_{\mathrm{cr}}$ to be inversely proportional to $\omega$, as originally suggested by Jongschaap et al. [5].

The argument above of course hinges crucially on the assumption that the present stress is determined by the history of strain. If, on the contrary, one chooses to postulate that the present stress is determined by the history of strain rates, a different conclusion would be reached. In fact, since the strain rate in an oscillatory motion is proportional to $\psi \omega$, the conclusion would be drawn that $\psi_{\mathrm{cr}}$ is inversely proportional to $\omega$ at all values of $\omega$, as indeed some constitutive equations would predict.

As quoted in the introduction, the experimental evidence strongly supports the conclusion that $\psi_{\mathrm{cr}}$ is independent of $\omega$. The fact that the low-frequency behavior ( $\psi_{\mathrm{cr}} \omega=$ constant) has only been reported in one case [5] is probably due to the fact that all experiments have been carried out at comparatively high frequencies with materials endowed with rather large values of $\Lambda$, say under conditions where $\omega \Lambda \gg 1$. 


\section{Theory}

Consider an incompressible viscoelastic fluid, and let $F^{t}(s)$ be the deformation gradient carrying the configuration at time $t-s$ into the one at time $t$ :

$\mathrm{d} X(t)=F^{t}(s) \cdot \mathrm{d} X(t-s)$,

where $\mathrm{d} X$ is the displacement vector between two material points. The relative strain $G^{t}(s)$ is defined as:

$G^{t}(s)=F^{t}(s)^{T} \cdot F^{t}(s)-1$.

By definition, $G^{t}(0)=0$. A simple fluid is one where the extra stress at time $t$ at a point is determined by the history of the relative strain at that point, i.e. by the tensor-valued function $G^{t}(s)$ over $s \in(0, \infty)$ :

$\tau(t)=\mathscr{F}\left\{G^{t}(s)\right\}$.

The concept of fading memory is formalized in the following way. First, a topology is assigned to the space of strain histories, such that, say, two histories are "close" to each other if they differ only in the distant past; second, the functional $\mathcal{F}\{\cdot\}$ in eqn. (3) is assumed to enjoy certain properties of smoothness with respect to the assigned topology.

Several different ways of performing Step 1 have appeared in the literature, see e.g. [6,7]; attention is here focused on the one presented by Coleman and Noll $[1,8]$ though most of the arguments would apply also to other forms.

A norm $\|\cdot\|$ for the strain histories is introduced:

$\left\|G^{t}(s)\right\|=\left[\int_{0}^{\infty}\left[h(s)\left|G^{t}(s)\right|\right]^{2} \mathrm{~d} s\right]^{1 / 2}$,

where $h(s)$ is an "influence function" (not to be confused with the memory function of linear viscoelasticity) which goes to zero fast enough when $s \rightarrow \infty$. The norm $\|\cdot\|$ induces a topology in the space of strain histories, since two histories $G_{1}^{t}(s)$ and $G_{2}^{t}(s)$ are recognized as being "close" when the norm of their difference is "small". Only this topology is, in fact, made use of in the subsequent theory; consequently, the influence function $h(s)$ is not uniquely determined by the functional $\mathcal{F}\{\cdot\}$, since different influence functions can induce the same topology. In other words, $h(s)$ cannot be considered a material function for the material described by the functional $\mathscr{F}\{\cdot\}$; and any argument to be developed must rely only on the topology induced by the norm $\|\cdot\|$, and not on any specific analytic form of the influence function $h(s)$.

If the material considered has always been kept at rest, the strain history is:

$G^{t}(s)=0^{\dagger}(s)$,

where $0^{+}(s)$ is the function mapping all $s \in[0, \infty)$ into the zero tensor. An incompressible fluid which has always been kept at rest exhibits a zero extra 
stress, say:

$0=\mathcal{F}\left\{0^{\dagger}(s)\right\}$.

If $\mathcal{F}\{\cdot\}$ is assumed to be twice Fréchet-differentiable at the rest history, one may write:

$\tau(t)=\delta \mathscr{F}\left\{0^{\dagger}(s) \mid G^{t}(s)\right\}+\mathscr{R}$,

where $\delta \mathscr{F}$ is linear in $G^{t}(s)$ (and can therefore be represented with the classical form of linear viscoelasticity), and the residual $R$ is of second order in the norm of $G^{t}(s)$ :

$|R|=O\left(\left\|G^{t}(s)\right\|^{2}\right)$

Now consider an oscillatory motion of amplitude $\psi$ and frequency $\omega$. For such a motion, the magnitude of the relative strain $\left|G^{t}(s)\right|$ has the following form:

$\left|G^{t}(s)\right|=\psi f(\omega s)$,

where the function $f(\cdot)$ has non-negative values of order unity, and enjoys the following two properties:

$f(0)=0$,

$f(\omega s+2 \pi)=f(\omega s)$.

Referring to the classification of oscillatory motions of Astarita and Marrucci [9], for Lagrangian-periodic flows $f(\cdot)$ is independent of $t$, while it is not for Eulerian-periodic flows; in both cases, it enjoys the stated properties.

The average value of $f(\cdot)$ over a cycle:

$\vec{f}=\frac{\omega}{2 \pi} \int_{s_{0}}^{s_{0}+2 \pi / \omega} f(\omega s) \mathrm{d} s=\frac{1}{2 \pi} \int_{0}^{2 \pi} f(\xi) \mathrm{d} \xi$,

is independent of both $s_{0}$ and $\omega . \bar{f}$ may depend in general on $\psi$, but is of order unity when $\psi$ tends to zero. Moreover, an easy calculation shows that, for small values of the argument,

$f(\omega s)=K \omega s+\mathrm{O}\left(\omega^{2} s^{2}\right)$,

where the constant $K$ can be made equal to unity by appropriate definition of $\psi$.

With that much background, the following results are immediately obtained. At very high frequencies, one has from eqns. (4) and (12):

$\lim _{\omega \rightarrow \infty}\left\|G^{t}(s)\right\|=\left[\int_{0}^{\infty}[h(s) \psi \bar{f}]^{2} \mathrm{~d} s\right]^{1 / 2}=\psi \bar{f}\left\|1^{\dagger}(s)\right\|$,

where $1^{\dagger}(s)$ is the function mapping all $s \in[0, \infty)$ into the unit tensor. 
At very low frequencies, consideration of eqns. (4) and (13), of the Schwarz inequality which, of course, holds for the norm $\|\cdot\|$, and of the retardation theorem of Coleman and Noll [8] yields:

$o(\omega)+\left\|G^{t}(s)\right\|=\left[\int_{0}^{\infty}[h(s) K \psi \omega s]^{2} \mathrm{~d} s\right]^{1 / 2}=K \psi \omega\|\underset{\approx}{\approx}\|$,

where $\lim _{\omega \rightarrow 0} o(\omega) / \omega=0$.

Considering eqn. (7), one realizes that the maximum allowable strain for linear viscoelasticity theory to hold is the value of $\psi$ for which the magnitude of the residual $\mathcal{R}$ becomes equal to some preassigned (small) positive number $\epsilon$. Hence, in view of the results above:

$\lim _{\omega \rightarrow \infty}|R|=O\left(\psi^{2}\right)$,

$\lim _{\omega \rightarrow 0}|R|=O\left(\psi^{2} \omega^{2}\right)$,

which show that the condition $|R|=\epsilon$ is fulfilled, at high frequencies, at some upper limit $\psi_{\mathrm{cr}}$ of the amplitude of strain which is independent of $\omega$; while at low frequencies $\psi_{\mathrm{cr}}$ is inversely proportional to $\omega$. Hence, the intuitive result given in the preceding section has been proved formally. It may be worth while to point out that the result for $\omega \rightarrow 0$ in eqn. (17) only applies to frequencies which are low enough for the series expansion in eqn. (13) to be truncated at the first term; this is also the condition for Newtonian behavior to be observed [8]. Hence, the inverse proportionality of $\psi_{\mathrm{cr}}$ and $\omega$ is expected to hold only at frequencies so low that linear viscoelasticity degenerates into its $\omega \rightarrow 0$ asymptote (the stress is in phase with the rate of strain); it is not surprising therefore, that such behavior has almost never in fact been observed experimentally.

\section{Comparison with previous analyses}

Previous analyses of the problem considered here have been presented by Marrucci and Astarita [2], Gross and Maxwell [3] and Jongschaap et al. [5]. Marrucci and Astarita essentially obtained by a rather simple argument the result given by eqn. (16), but failed to notice that it only holds at sufficiently high frequencies. Both Gross and Maxwell, and Jongschaap et al. considered two specific constitutive equations, namely, the Bird-Carreau model [10] and Tanner's model of network rupture [11], and have reached the same conclusions: namely, that the Bird-Carreau model predicts $\psi_{\mathrm{cr}}$ to be inversely proportional to $\omega$ at all frequencies, while Tanner's model predicts it to be independent of $\omega$ at all frequencies.

The first conclusion is not surprising. The Bird-Carreau model does not describe a simple fluid enjoying the smoothness properties discussed in the 
preceding section; explicit dependency of the parameters of the model on the strain rate places it in the category discussed by Marrucci and Astarita [2], and, hence, the model does not degenerate into linear viscoelasticity as a simple fluid with fading memory. Experimental results belie this prediction of the Bird-Carreau model.

The second conclusion is, at first sight, a paradox, since Tanner's model is a special case of a simple fluid with fading memory, and it should thus obey both eqns. (16) and (17), and not eqn. (16) at all values of $\omega$. In fact, that is exactly what Tanner's model does, although this has not been recognized in [3] and [5]. The fact is that, in addition to the network-rupture hypothesis, Tanner's model has a memory function which is a sum of exponentials. When the frequency $\omega$ becomes much less than the inverse of the largest relaxation time $\lambda_{\max }$, Tanner's model degenerates into linear viscoelasticity even if the amplitude of strain $\psi$ is larger than the one corresponding to network rupture, provided $\omega \lambda_{\max } \psi$ is smaller; hence, the result embodied in eqn. (17) is recovered. Again, the required frequencies are exceedingly low, and, in fact, the result embodied in eqn. (16) is presumably valid over the usual experimental range of frequencies.

Jongschaap et al. also consider the general case of a simple fluid with fading memory, but they choose'to assign a specific form to the influence function $h(s)$, say:

$h(s)=\exp (-\alpha s)$.

They then obtain the two asymptotic results for $\omega>>\alpha$ and for $\omega<<\alpha$, respectively. The analysis suffers from the fact that, since $h(s)$ is not a material function, $\alpha$ is not a material parameter, and therefore comparison of $\omega$ with $\alpha$ is, strictly speaking, meaningless. Of course, in view of eqns. (16) and (17), a "relaxation time" could be extracted from experimental data as the inverse of the frequency at which the extrapolated asymptotes cross each other in a $\log \psi_{\mathrm{cr}}$ versus $\log \omega$ plot; yet there is no a priori reason why such a relaxation time should be independent of the form of the function $f(\cdot)$.

\section{References}

1 B.D. Coleman and W. Noll, Foundations of linear viscoelasticity, Rev. Mod. Phys., 33 (1961) 239; Erratum, ibid., 36 (1964) 1103.

2 G. Marrucci and G. Astarita, Comments on the validity of a common category of constitutive equations, Rheol. Acta, 13 (1974) 754.

3 L.H. Gross and B. Maxwell, The limit of linear viscoelastic response in polymer melts as measured in the Maxwell orthogonal rheometer, Trans. Soc. Rheol., 16 (1972) 577.

4 W. Philippoff, Vibrational measurements at large amplitudes, Trans. Soc. Rheol., 10 (1962) 317.

5 R.J.J. Jongschaap, K.H. Knapper and J.S. Lopulissa, About the limit of linear viscoelastic response in the flow between eccentric rotating disks, Joint Meeting $\mathrm{Br}$., Ital. and Neth. Socs. Rheol., Pisa, 1977.

6 B.D. Coleman and V.J. Mizel, Norms and semigroups in the theory of fading memory, Arch. Ration. Mech. Anal., 23 (1966) 87. 
7 C. Truesdell and W. Noll, The non-linear field theories, in Encycl. of Phys., Vol. III/3, Springer-Verlag, Berlin, 1965.

8 B.D. Coleman and W. Noll, An approximation theorem for functionals, with applications in continuum mechanics, Arch. Ration. Mech. Anal., 6 (1960) 355.

9 G. Astarita and G. Marrucci, Principles of Non-Newtonian Fluid Mechanics, McGraw Hill, Maidenhead, 1974.

10 R.B. Bird and P.J. Carreau, A non-linear viscoelastic model for polymer solutions and melts, Chem. Eng. Sci., 23 (1968) 427.

11 R.I. Tanner, Network rupture and the flow of concentrated polymer solutions, AIChE J., 15 (1969) 177. 